\title{
Duration Dependence in Bull and Bear Stock Markets
}

\author{
Haigang Zhou, Steven E. Rigdon \\ ${ }^{1}$ Department of Finance, Cleveland State University, Cleveland, USA \\ ${ }^{2}$ Department of Mathematics and Statistics, Southern Illinois University Edwardsville, Edwardsville, USA \\ E-mail: H.zhou16@csuohio.edu
}

Received January 20, 2011; revised March 15, 2011; accepted April 1, 2011

\begin{abstract}
Testing duration in stock markets concerns the ability to predict the turning points of bull and bear cycles. The Weibull renewal process has been used in previous studies to analyze duration dependence in economic and financial cycles. A goodness-of-fit test, however, shows that this model does not fit data from U.S. stock market cycles. As a solution, this study fits the modulated power law process that relies on less restrictive assumptions. Moreover, it measures both the long term properties of bull and bear markets, such as the tendency of the cycles to become shorter (or longer), as well as the short term effects, such as duration dependence. The results give evidence of negative duration dependence in all samples of bull markets and evidence of positive duration dependence in complete, peacetime and post WWII samples of bear markets. There is no evidence of any structural change in duration dependence after WWII in either bull or bear markets. The results show that bull and bear markets tend to get progressively shorter, but for bull markets this trend has accelerated since WWII whereas for bear markets this trend has decelerated since WWII. Goodness-of-fit tests suggest that the modulated power is a suitable model for U.S. stock market cycles.
\end{abstract}

Keywords: Modulated Power Law Process, Business Cycles, Financial Cycles, Power Law Process, Weibull Distribution, Renewal Process

\section{Introduction}

The duration dependence of stock market cycles can help to pinpoint the peaks and troughs in these cycles. The predictability of turning points and the relevance of duration dependence analysis in financial markets has been studied in [1] and [2]. Unstructured statistical models have been used in modeling duration dependence in business cycles [3], REOIT cycles [1], and stock market cycles [2] and [4].

Previous studies have often used the Weibull renewal process to study duration dependence in business and financial cycles. For the Weibull renewal process, the probability of an event in a small interval depends only on the time since the previous event, and not on the previous pattern of failures or the times since the process initially began. In particular, this model assumes that after the occurrence of an event, the system is always in exactly the same condition, precluding the possibility of a long term change in the system. Through goodness-offit tests, it was shown in [4] that U.S. business cycles do not fit the simple Weibull renewal process model.

The nonhomogeneous Poisson process (NHPP) is ano- ther model that has been used to odel the occurrence of events in time. For an NHPP, the probability of an event in a small interval is some function of time since the initial startup of the system. An event and the subsequent restarting of the system, therefore, has no effect on the system performance. If the probability of an event occurring in a small interval is constant across time, then the process is a homogeneous Poisson process where the times between events are independent and identically distributed exponential random variables. This special case is also a renewal process.

Thus, for a renewal process, the system starts anew each time there is an event, whereas for the NHPP, the process picks up right where it left off. In the reliability context, the renewal process is described as a good-as-new, or same-as-new model, and the NHPP is described as a bad-as-old or same-as-old model. Therefore, a renewal process can model duration dependence but not any long term effects, such as the tendency of intervals to get longer or shorter. The NHPP, on the other hand, can model long term effects, but not duration dependence.

We propose using the modulated power law process 
(MPLP) to model duration dependence for U.S. stock market cycles. This model, suggested by [5,6], and [7], is a compromise between a renewal process and an NHPP. With the MPLP, we are able to estimate long term effects, such as events becoming progressively more (or less) frequent, as well as short term effects, such as duration dependence.

Our study is also related to the Frisch-Slutsky paradigm of cycles. Frisch [8] and Slutsky [9] state that there is no need to appeal to specific determinant causes of cycles. Many of the advances in theories of cyclical volatilities are in the field of business cycles and little theoretical work has been devoted to financial cycles. Although much of the following discussion on theories of cycles are from business cycles, we believe that the insight from the theories can also improve our understanding of financial cycles, even without identifying the exact sources of shocks to the financial markets. Much progress has been made in understanding business cycles and even economists are not able to agree on the causes of cyclical volatility. The exact causes of cyclical volatility are debated and identifying the sources of shocks to the financial markets is beyond the scope of this study. [10] provides a detailed review on the evolution of business cycle theories. References [11] and [12] provide a framework to identify shocks to the financial sectors. Frisch [8] and Slutsky [9] argued that many phenomena existed that could precipitate a real shock in the market's equilibrium path. A large negative shock, although rare, would be sufficient to draw the average market activity away from the equilibrium level for a sufficiently long period of time to be considered a downswing. Slutsky argued that "clusters" of small negative shocks can also move the market away from its equilibrium. As we describe in Section 2.2, the MPLP involves one parameter which can be thought of as the accumulated number of shocks.

We fit the modulated power law process do data from bull and bear markets. We consider separately peacetime and war time data, as well as pre- and post-WWII data. We find evidence of negative duration dependence in all samples of bull markets and in pre-WWII bear markets, and evidence of positive duration dependence in complete, peacetime, and post-WWII samples of bear markets. In regards to the long term effect, evidence shows that bull and bear markets tend to get progressively shorter, but for bull markets, this trend has accelerated since WWII, whereas for bear markets, this trend has decelerated since WWII.

Section 2 describes the methodology used in the study as well as the sampling data. Section 3 presents descriptive statistics and empirical results, while Section 4 offers concluding remarks.

\section{Methodology and Data}

In this study, both the Weibull renewal process and the MPLP are used to examine the duration of bull and bear markets. The Weibull renewal process and its variations are widely used in the duration dependence literature. The Weibull renewal process assumes a linear relationship between the log of the intensity function (measured from the last event and restart of the system) and that of the durations. The MPLP is a generalization of both the Weibull renewal process and the NHPP with a power law intensity function. When we consider bull markets, we ignore the intervening bear markets and treat the interevent times as if they were back-to-back. Bear markets are treated similarly.

\subsection{Weibull Renewal Process}

The Weibull renewal process assumes that the times between events are independent random variables, each with the same Weibull distribution. The probability density function (PDF) and the hazard function for the Weibull distribution are

$$
f(t)=\frac{\kappa}{\theta}\left(\frac{t}{\theta}\right)^{\kappa-1} \exp \left(-\left(\frac{t}{\theta}\right)^{\kappa}\right), t>0,
$$

and

$$
h(t)=\frac{f(t)}{1-F(t)}=\frac{\kappa}{\theta}\left(\frac{t}{\theta}\right)^{\kappa-1}, t>0 .
$$

Here $\kappa>0$ is a shape parameter and $\theta$ is a scale parameter. The hazard function is increasing when $\kappa>1$, decreasing when $\kappa<1$, and constant when $\kappa=1$. When $\kappa=1$, the Weibull distribution reduces to the exponential distribution.

An increasing hazard function $(\kappa>1)$ implies that the conditional probability of a turning point in the market cycle will thus increase as the duration of the cycle increases; this indicates positive duration dependence. On the other hand a decreasing hazard function $(\kappa<1)$ implies that the conditional probability of a turning point will decrease as the duration of the cycle increases; this indicates negative duration dependence.

We fit the Weibull renewal process to the bull and bear markets from 1885 to 2000. Goodness-of-fit tests reject the hypothesis of a Weibull renewal process for both the bull markets and the bear markets. Further details regarding the analysis of these data are found in Section 3.2.

\subsection{The Modulated Power Law Process}

The MPLP was introduced by [5] and [6] as a compro- 
mise between the Weibull renewal process and an NHPP with intensity function

$$
\lambda(t)=\frac{\kappa}{\theta}\left(\frac{t}{\theta}\right)^{\kappa-1}, \quad t>0,
$$

where time $t$ is measured from the initial startup of the system. The NHPP with this parametric form of the intensity function is called the power law process in the reliability literature. As we mentioned in Section 1, the Weibull renewal process can be thought of as a same-as-new model, whereas the NHPP is a same-as-old model because of the assumptions about what happens after an event and restart of the system.

The MPLP is then derived as follows. Suppose that shocks occur according to the power law process, the NHPP with intensity function given in (1). Suppose further that an event does not occur at every shock, but rather after every $\beta$ shocks. For now we assume that $\beta$ is a positive integer. After the $\beta$ th shock, the shock counter is reset to zero, but time $t$ is not reset to zero. This is what allows us to model the long term effects, such as the tendency of times between events to increase. For example, suppose that $\kappa=2$ and $\beta=5$; then we would observe that shocks occur more and more frequently as time advances, because the intensity function for the shocks is proportional to $t^{2-1}=t$, an increasing function in $t$. However, each time an event occurs, the shock counter would be reset, so we would have positive duration dependence. Thus, we would have positive duration dependence while the long term tendency is to have shorter durations. Larger values of $\beta$ indicate stronger positive duration dependence, whereas larger values of $\kappa$ indicate a long term tendency for shorter and shorter intervals between events.

Since we consider the bull markets (and also the bear markets) as if they were back-to-back, we let $T_{i}$ denote the time of the $i$ th event, measured from the initial point of data collection. We define $T_{0}=0$. The interevent times, $X_{i}=T_{i}-T_{i-1}$, then represent the length of each bull (or bear) market.

It can be shown (see, for example, [6,7]) that the random variables

$$
X_{i}=\left(\frac{T_{i}}{\theta}\right)^{\kappa}-\left(\frac{T_{i-1}}{\theta}\right)^{\kappa}, \quad i=1,2, \cdots, n,
$$

are independent and identically distributed random variables having a gamma distribution with PDF

$$
f(x \mid \beta)=\frac{x^{\beta-1}}{\Gamma(\beta)} \exp (-x), \quad x>0 .
$$

From this result, we can determine the likelihood function for the $X_{i}$ 's, and then the likelihood function for the observed $T_{i}$ 's. Note that the $T_{i}$ 's are the event times measured from the initial startup of the system. The log likelihood function is then

$$
\begin{aligned}
\ell\left(\theta, \kappa, \beta \mid t_{1}, t_{2}, \cdots, t_{n}\right) & =-\left(\frac{t_{n}}{\theta}\right)^{\kappa}+n \ln \kappa-n \ln \Gamma(\beta) \\
& -n \kappa \beta \ln \theta+(\kappa-1) \sum_{i=1}^{n} \ln t_{i} \\
& +(\beta-1) \sum_{i=1}^{n} \ln \left(t_{i}^{\kappa}-t_{i-1}^{\kappa}\right)
\end{aligned}
$$

The maximum likelihood estimates can then be obtained by differentiating the log-likelihood function with respect to each parameter, setting the results equal to zero, and using a numerical method to approximate the solution.

The description above relies on counting the number of shocks to the system, requiring that $\beta$ be a positive integer. However, the likelihood in (2) is a valid likelihood for all positive values of $\beta$, not just for positive integers. The following example suggests how noninteger values can be allowed for $\beta$. Suppose that local events occur at the rate $\lambda(t)$. Each time a local event occurs, the probability that it is a shock to the system is $1 / 2$. Suppose also, that $\beta=5$ shocks will cause a system event (end of bull/bear market). Since only about half of the local events will cause a shock, there will be on average $(1 / 2) \times 5=2.5$ shocks that cause a failure. The model just described is indistinguishable from a MPLP with rate $\lambda(t)$ and $\beta=2.5$. Other fractional values can be similarly explained. In looking at another way of explaining fractional values of $\beta$, [7] simulated a number of processes for various values of $\beta$. They found that with large values of $\beta$, the event times were evenly spaced, possibly with a long term effect of interevent times getting shorter or longer. Thus for large $\beta$, as the duration gets longer, we get closer to the next point in the spacing, which means that the conditional probability of a system event gets larger. For $\beta<1$, the events are very much clustered, with a few very short interevent times and a few very long interevent times. These are much more clustered than would be expected by a Poisson process. The existence of clustering suggests negative duration dependence, since as the duration increases, it becomes more likely that it is one of the very long interevent times. For a third way of considering fractional values of $\beta$, we consider special cases of the MPLP. When $\beta=1$, the MPLP with parameters $\kappa$ and $\theta$ is a NHPP with intensity function $\lambda(t)=$ $(\theta / \kappa)(t / \theta)^{\kappa-1}, t>0$. When $\kappa=1$, then the MPLP with parameters $\beta$ and $\theta$ is a Weibull renewal process. Since noninteger values of $\beta$ are allowed in the Weibull distribution, it seems reasonable to allow noninteger values in a generalization of the Weibull renewal process. 
Finally, if $\kappa=\beta=1$, then the MPLP reduces to the homogeneous Poisson process with intensity function $\lambda(t)=1 / \theta$.

\subsection{Data}

Data from stock market cycles exist as far back as 1885 . These data, taken from [13], are reproduced in Table 1. The bull and bear markets during wars are indicated in bold face. Figure 1 shows plots of durations for bull and bear markets both pre- and post-WWII.

\section{Empirical Findings}

\subsection{Descriptive Statistics}

Descriptive statistics of bull and bear markets, broken down into peacetime, pre-WWII, and post-WWII are reported in Table 2 . The complete sample includes all observations, while the peacetime sample excludes war-time cycles. The average duration of bull markets is 28 months for the complete sample, and it is 27 months for the peacetime bull markets. The average duration of bear markets is 15 months for both complete and peace time samples. The results indicate that war does not have significant impact on the duration of stock markets. This differs from the results reported in [4] that the average length of the complete sample of expansions is lower than that of peace time expansions. We also observe that the average duration of bull markets is longer and the average duration of bear markets is shorter in the post-WWII subsample than in the pre-WWII subsample.

\subsection{Empirical Results from the Weibull Analysis}

Table 3 shows the maximum likelihood estimates of the parameters, along with confidence intervals. For bull markets, the MLE for $\kappa$ is $\hat{\kappa}=1.836$ for the complete sample. Because the $95 \%$ confidence interval excludes 1 , we conclude that there is evidence that the true value of $\kappa$ exceeds 1 . This indicates that positive duration dependence exists in cycles of bull markets. Similar results are obtained for the peace time, and pre- and post-WWII data (both bull and bear markets), supporting the existence of positive duration dependence. This implies that the probability of a bull/bear market ending increases as the duration increases. These results differ from those

Table 1. U.S. bull and bear stock markets (1885 through 2000). Dates of peaks and troughs in the U.S. stock markets. Durations (in months) are also shown. Data are obtained from [13]. Bold face indicates wartime bull and bear markets.

\begin{tabular}{|c|c|c|c|c|c|c|c|}
\hline \multicolumn{4}{|c|}{ Pre-WWII } & \multicolumn{4}{|c|}{ Post- WWII } \\
\hline Trough & Peak & Bull & Bear & Trough & Peak & Bull & Bear \\
\hline Jan-1885 & May-1887 & 28 & 13 & & May-1946 & & 21 \\
\hline Jun-1888 & May-1890 & 23 & 7 & Feb-1948 & Jun-1948 & 4 & 12 \\
\hline Dec-1890 & Aug-1892 & 20 & 31 & Jun-1949 & Jan-1953 & 43 & 9 \\
\hline Mar-1895 & Sep-1895 & 6 & 11 & Oct-1953 & Jul-1956 & 33 & 17 \\
\hline Aug-1896 & Apr-1899 & 32 & 17 & Dec-1957 & Jul-1959 & 19 & 15 \\
\hline Sep-1900 & Sep-1902 & 24 & 13 & Oct-1960 & Dec-1961 & 14 & 6 \\
\hline Oct-1903 & Sep-1606 & 35 & 14 & Jun-1962 & Jan-1966 & 43 & 9 \\
\hline Nov-1907 & Dec-1909 & 25 & 7 & Oct-1966 & Dec-1968 & 26 & 18 \\
\hline Jul-1910 & Sep-1912 & 26 & 27 & Jun-1970 & Jan-1973 & 31 & 23 \\
\hline Dec-1914 & Nov-1916 & 23 & 13 & Dec-1974 & Sep-1976 & 21 & 18 \\
\hline Dec-1917 & Jul-1919 & 19 & 25 & Mar-1978 & Dec-1980 & 33 & 19 \\
\hline Aug-1921 & Mar-1923 & 19 & 7 & Jul-1982 & Jun-1983 & 11 & 11 \\
\hline Oct-1923 & Sep-1929 & 71 & 33 & May-1984 & Aug-1987 & 39 & 3 \\
\hline Jun-1932 & Feb-1934 & 20 & 13 & Nov-1987 & May-1990 & 30 & 5 \\
\hline Mar-1935 & Feb-1937 & 23 & 14 & Oct-1990 & Jan-1994 & 39 & 5 \\
\hline Apr-1938 & Oct-1938 & 6 & 42 & Jun-1994 & Aug-2000 & 74 & \\
\hline Apr-1942 & May-1946 & 49 & & & & & \\
\hline
\end{tabular}




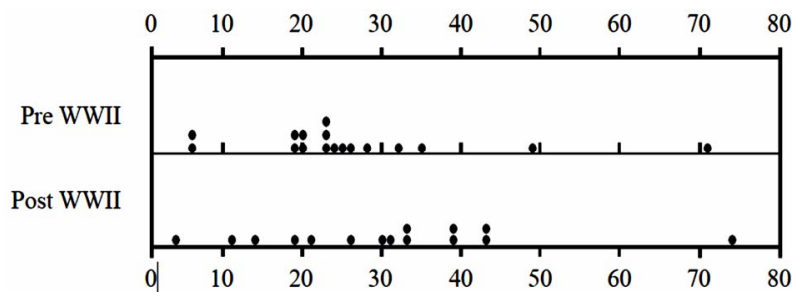

(a)

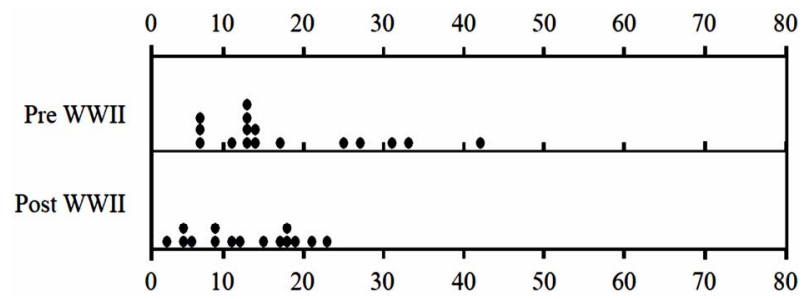

(b)

Figure 1. Dot plots for durations of bull and bear markets, measured in months. Multiple occurrences are indicated by stacking the dots. (a) Bull market; (b) Bear market.

Table 2. Descriptive statisticsof bull and bear markets. The complete sample includes all bull and bear markets from January 1885 to August 2000, including separate statistics for peace time, pre-WWII and post-WWII.

\begin{tabular}{|c|c|c|c|c|}
\hline & Complete & Peace Time & Pre-WWII & Post-WWII \\
\hline \multicolumn{5}{|c|}{ Bull Markets } \\
\hline Mean & 28 & 27 & 26 & 31 \\
\hline Median & 26 & 24 & 23 & 31 \\
\hline Std. Dev. & 15.84 & 16.50 & 15.15 & 16.82 \\
\hline Skewness & 1.21 & 1.54 & 1.68 & 0.91 \\
\hline Kurtosis & 2.25 & 3.22 & 4.25 & 2.20 \\
\hline \multicolumn{5}{|c|}{ Bear Markets } \\
\hline Mean & 15 & 15 & 18 & 13 \\
\hline Median & 13 & 14 & 18 & 18 \\
\hline Std. Dev. & 9.01 & 8.07 & 10.47 & 6.45 \\
\hline Skewness & 1.14 & 0.95 & 1.05 & 6.45 \\
\hline Kurtosis & 1.36 & -0.15 & 0.21 & -1.37 \\
\hline
\end{tabular}

reported in [2] who report the existence of duration dependence in post-WWII bear markets and in pre-war bull markets, but they found no evidence of duration dependence in pre-war bear markets and post-war bull markets.

The Weibull renewal process implies that the process is renewed after every event. This precludes any longterm effects, such as the tendency for the intervals to become shorter or longer. We tested the adequacy of the
Weibull distribution using goodness-of-fit statistics proTable 3. MLEs and confidence intervals. Maximum likelihood estimates and confidence intervals for the parameters of the Weibull renewal process.

\begin{tabular}{|c|c|c|c|}
\hline & \multicolumn{3}{|c|}{ 95\% Confidence Interval for $\kappa$} \\
\hline & $\hat{\kappa}$ & Lower & Upper \\
\hline \multicolumn{4}{|c|}{ Bull Markets } \\
\hline Complete & 1.865 & {$[1.512$,} & 2.226 ] \\
\hline Peace time & 1.699 & {$[1.369$,} & $2.108]$ \\
\hline Pre-WWII & 1.900 & {$[1.505$,} & $2.398]$ \\
\hline Post-WWII & 1.787 & {$[1.306$,} & $2.444]$ \\
\hline \multicolumn{4}{|c|}{ Bear Markets } \\
\hline Complete & 1.853 & {$[1.547$,} & $2.219]$ \\
\hline Peace time & 1.993 & {$[1.629$, } & $2.437]$ \\
\hline Pre-WWII & 1.908 & {$[1.487$,} & $2.448]$ \\
\hline Post-WWII & 2.187 & {$[1.660$,} & $2.880]$ \\
\hline \multicolumn{4}{|c|}{ 95\% Confidence Interval for $\theta$} \\
\hline & $\hat{\theta}$ & Lower & Upper \\
\hline \multicolumn{4}{|c|}{ Bull Markets } \\
\hline Complete & 32.30 & {$[32.07$,} & $32.54]$ \\
\hline Peace time & 31.08 & {$[30.82$,} & 31.33] \\
\hline Pre-WWII & 29.83 & {$[29.53$,} & $30.16]$ \\
\hline Post-WWII & 35.22 & {$[34.86$,} & $35.58]$ \\
\hline \multicolumn{4}{|c|}{ Bear Markets } \\
\hline Complete & 17.44 & {$[17.22$,} & 17.67] \\
\hline Peace time & 16.84 & {$[16.60$,} & 17.09] \\
\hline Pre-WWII & 20.36 & {$[20.06$,} & 20.68] \\
\hline Post-WWII & 14.40 & {$[14.08$,} & 14.73] \\
\hline
\end{tabular}

posed by [14]. Table 4 shows the various goodness-of-fit tests that we applied. The upper part of Table 4 reports the test results for bull markets, while the bottom part reports those for bear markets. For the complete sample, all three tests statistics reject the null hypothesis that the Weibull is the distribution for the interevent times. Both the Cramér-von Mises $W^{2}$ and Watson $U^{2}$ tests reject the null hypothesis at the $1 \%$ level, while the Anderson-Darling $A^{2}$ test rejects at the $10 \%$ significance level. Similar results are reported for the peace time and pre-and post-war bull markets.

For bear markets, the Cramér-von Mises and Watson tests both reject the null hypothesis that the Weibull dis- 
tribution is adequate at the $1 \%$ significance level. TheAnderson-Darling statistics fail to reject the null hyTable 4. Goodness-of-fit tests for the Weibull renewal process. Goodness-of-fit tests for the Weibull renewal process for bull markets (upper) and bear markets (lower), using the complete sample, peace time sample, and pre-WWII and post-WWII. All statistics are adjusted by multiplying by $1+0.2 / \sqrt{n}$. The corresponding $P$-values are given in parentheses.

\begin{tabular}{|c|c|c|c|c|}
\hline & Complete & Peace Time & Pre-WWII & Post-WWII \\
\hline \multicolumn{5}{|c|}{ Bull Markets } \\
\hline $\begin{array}{l}\text { Cramer-von } \\
\text { Mises } W^{2}\end{array}$ & $\begin{array}{c}5.74 \\
(<0.01)\end{array}$ & $\begin{array}{c}4.92 \\
(<0.01)\end{array}$ & $\begin{array}{c}3.21 \\
(<0.01)\end{array}$ & $\begin{array}{c}2.71 \\
(<0.01)\end{array}$ \\
\hline Watson $U^{2}$ & $\begin{array}{c}5.73 \\
(<0.01)\end{array}$ & $\begin{array}{c}4.92 \\
(<0.01)\end{array}$ & $\begin{array}{c}3.21 \\
(<0.01)\end{array}$ & $\begin{array}{c}2.71 \\
(<0.01)\end{array}$ \\
\hline $\begin{array}{l}\text { Anderson- } \\
\text { Darling } A^{2}\end{array}$ & $\begin{array}{l}0.638 \\
(>0.1)\end{array}$ & $\begin{array}{l}0.656 \\
(>0.1)\end{array}$ & $\begin{array}{c}0.957 \\
(0.025)\end{array}$ & $\begin{array}{c}0.366 \\
(>0.25)\end{array}$ \\
\hline \multicolumn{5}{|c|}{ Bear Markets } \\
\hline $\begin{array}{l}\text { Cramer-von } \\
\text { Mises } W^{2}\end{array}$ & $\begin{array}{c}5.79 \\
(<0.01)\end{array}$ & $\begin{array}{c}4.74 \\
(<0.01)\end{array}$ & $\begin{array}{c}3.23 \\
(<0.01)\end{array}$ & $\begin{array}{c}2.63 \\
(<0.01)\end{array}$ \\
\hline Watson $U^{2}$ & $\begin{array}{c}5.79 \\
(<0.01)\end{array}$ & $\begin{array}{c}4.74 \\
(<0.01)\end{array}$ & $\begin{array}{c}3.22 \\
(<0.01)\end{array}$ & $\begin{array}{c}2.63 \\
(<0.01)\end{array}$ \\
\hline $\begin{array}{l}\text { Anderson- } \\
\text { Darling } A^{2}\end{array}$ & $\begin{array}{c}0.291 \\
(>0.25)\end{array}$ & $\begin{array}{c}0.2392 \\
(0.25)\end{array}$ & $\begin{array}{c}0.727 \\
(0.1)\end{array}$ & $\begin{array}{c}0.427 \\
(>0.25)\end{array}$ \\
\hline
\end{tabular}

pothesis at the $10 \%$ significance level for the complete, peace time and post-war samples.

In summary, several goodness-of-fit tests show that the Weibull renewal process is not adequate for studying the duration dependence in bull and bear markets. As we discuss in the next section, the issue of goodness-of-fit testing is not whether the Weibull is better than some other distribution, such as the gamma, but rather, whether a nonstationary model, such as the NPLP is better than a stationary model, such as the Weibull renewal process.

\subsection{Empirical Results from the MPLP Analysis}

Next, we use the MPLP model to examine duration dependence in stock markets. The MPLP model allows long term effects, such as the interevent times to get shorter (or longer) in addition to short term effects like duration dependence. The MPLP contains three parameters: $\beta$, a parameter that affects duration dependence; $\kappa$, a parameter that affects the tendency of the interevent times to get shorter or longer; and $\theta$, a scale parameter. Table 5 reports maximum likelihood estimates of these three parameters; the upper part reports the results for bull markets, and the bottom part reports those for bear markets.

The estimate of $\beta$ for the complete sample of bull markets is 0.914 , indicating negative duration dependence in bull markets, i.e., the probability for a bull mar ket to end decreases as the duration of the bull market increases. The estimate for $\beta$ for the peace time data is Table 5. Point estimates and likelihood ratio tests for the MPLP parameters. Maximum likelihood estimates of $\beta$, $\kappa$, and $\theta$, results of likelihood ratio tests, and corresponding $\mathbf{P}$-values for testing whether the parameters equal 1.

\begin{tabular}{|c|c|c|c|c|}
\hline & Complete & Peace Time & Pre-WWII & Post-WWII \\
\hline \multicolumn{5}{|c|}{ Bull Markets } \\
\hline $\begin{array}{c}\hat{\beta} \quad \text {-value for } \\
H_{0}: \beta=1\end{array}$ & $\begin{array}{c}0.914 \\
\left(<10^{-4}\right)\end{array}$ & $\begin{array}{c}0.931 \\
\left(<10^{-4}\right)\end{array}$ & $\begin{array}{c}0.9587 \\
(0.0334)\end{array}$ & $\begin{array}{l}0.7701 \\
\left(<10^{-4}\right)\end{array}$ \\
\hline $\begin{array}{c}\hat{\kappa} \quad \text {-value for } \\
H_{0}: \kappa=1\end{array}$ & $\begin{array}{l}3.2319 \\
\left(<10^{-4}\right)\end{array}$ & $\begin{array}{c}2.885 \\
(0.0003)\end{array}$ & $\begin{array}{c}3.4536 \\
(0.0010)\end{array}$ & $\begin{array}{c}3.9204 \\
(0.0049)\end{array}$ \\
\hline $\begin{array}{c}P \text {-value for } \\
H_{0}: \beta=\kappa=1\end{array}$ & $(0.0001)$ & $(0.0018)$ & $(0.0060)$ & $(0.0049)$ \\
\hline$\hat{\theta}$ & 5.6811 & 6.8521 & 6.4177 & 2.3173 \\
\hline \multicolumn{5}{|c|}{ Bear Markets } \\
\hline $\begin{array}{c}\hat{\beta} \quad P \text {-value for } \\
H_{0}: \beta=1\end{array}$ & $\begin{array}{l}1.0654 \\
\left(<10^{-4}\right)\end{array}$ & $\begin{array}{l}1.0851 \\
\left(<10^{-4}\right)\end{array}$ & $\begin{array}{c}0.8793 \\
(0.0007)\end{array}$ & $\begin{array}{l}1.2414 \\
\left(<10^{-4}\right)\end{array}$ \\
\hline $\begin{array}{c}\hat{\kappa} \quad P \text {-value for } \\
H_{0}: \kappa=1\end{array}$ & $\begin{array}{l}3.1506 \\
\left(<10^{-4}\right)\end{array}$ & $\begin{array}{l}3.2843 \\
\left(<10^{-4}\right)\end{array}$ & $\begin{array}{c}3.8117 \\
(0.0021)\end{array}$ & $\begin{array}{c}3.6476 \\
(0.0012)\end{array}$ \\
\hline $\begin{array}{c}P \text {-value for } \\
H_{0}: \beta=\kappa=1\end{array}$ & $(0.0002)$ & $(0.0005)$ & $(0.0045)$ & $(0.0039)$ \\
\hline$\hat{\theta}$ & 6.484 & 6.4239 & 2.6766 & 7.601 \\
\hline
\end{tabular}

0.931 with similar implications.

Consider now the pre- and post-WWII data. The estimates of $\beta$ are $\hat{\beta}=0.9587$ for the pre-war sample, and $\hat{\beta}=0.7701$ for the post-war sample. Although the post-war estimate is lower than the pre-war estimate, both are statistically less than 1 . Therefore, evidence of negative duration dependence exists both before and after WWII. In summary, we find strong evidence of negative duration dependence in the samples of bull markets, indicating that the likelihood for a bull market to end shortly decreases as the length of a bull market increases.

The estimate of $\kappa$ for the complete sample of bull markets is $\hat{\kappa}=3.2319$, indicating that the long term effect is for the interevent times to become shorter. For peace time data, the estimate is $\hat{\kappa}=2.885$, indicating that the interevent eimes tend to get shorter, but at a rate that is less than overall. The estimates of $\kappa$ before and after WWII are, respectively, $\hat{\kappa}=3.4536$ and $\hat{\kappa}=3.9204$, indicating that the interevent times tend to get shorter at a faster rate over the post-WWII period than over the pre-WWII period. 
For the complete sample of bear markets, the estimate of $\beta$ is $\hat{\beta}=1.0654$, indicating positive duration dependence in bear markets. Therefore the probability that a bear market ends increases with the length of the bear market. This result is the opposite of that observed for bull markets. There does seem to be a difference between duration dependence in pre-war and post-war bear markets. For the pre-war data, the estimate is $\hat{\beta}=0.8793$ (negative duration dependence) and for the post-war data, the estimate is $\hat{\beta}=1.2414$ (positive duration dependence). Both are significantly different from 1.

The estimates of $\kappa$ exceed 3 for all cases considered and all are significantly different from 1 . These results are similar to those for bull markets and indicate that the cycles tend to become shorter over time. We also notice that $\hat{\kappa}$ is higher for the peace time sample than for the complete sample, indicating that wars slightly reduce the resilience of bear markets to external shocks. There is also a slight decrease in $\hat{\kappa}$ in the post-WWII sample than in the pre-WWII sample, indicating that the interevent times are getting shorter at a slower pace in the post-WWII period. Based on the Frisch-Slutsky paradigm, the lower $\hat{\kappa}$ indicates reduced resilience of bear markets to external shocks in the post-WWII period than in the pre-WWII period.

Table 5 also gives the results of a number of hypothesis tests. We test whether $\beta$ or $\kappa$ (or both simultaneously) are equal to 1 . For these parameters, the value of 1 is important; for $\beta$, the value $\beta=1$ is the boundary between negative and positive duration dependence, whereas for $\kappa$, the value $\kappa=1$ is the boundary between the cycles tending to get shorter or longer across time. All of the hypothesis tests reject the null hypothesis of the parameter being 1 .

In summary, there is evidence of negative duration dependence in all bull markets. There is evidence of positive duration dependence in all bear markets except those before WWII. Thus, bull markets tend to become stronger and bear markets weaker as the cycle lengthens.

In regards to the long-term effect, the results show that bull and bear markets tend to get progressively shorter, but for bull markets, this trend has accelerated since WWII, whereas for bear markets this trend has decelerated since WWII. Finally, since the lengths of bull and bear markets tend to get shorter over time, a stationary model such as the Weibull renewal process, or any renewal process for that matter, is inadequate to model the cycle times. A nonstationary model such as the MPLP is needed.

\section{Conclusions}

Possible models for the stochastic point process that go- verns financial cycles include the renewal process, the NHPP, and the MPLP. The MPLP is a generalization of both the renewal process and the NHPP in the sense that if $\beta=1$, then the MPLP reduces to the NHPP, and if $\kappa=1$, then the MPLP reduces to the Weibull renewal process. If both $\beta=1$ and $\kappa=1$, then the MPLP reduces to the homogeneous Poisson process.

Traditionally, the Weibull renewal process has been applied as a model for business and financial cycles. However, one of the assumptions implicitly made in the Weibull renewal analysis seems dubious. The Weibull renewal process also assumes that the underlying stochastic process does not change across time. In other words, a new market (i.e., a market that has just changed from bull to bear, or vice-versa) is the same now as any new market in the past. Considering that data come from such a long period (1885 to 2000), this assumption appears unreasonable. A model is needed that can account for both duration dependence and log term trends for cycles to become shorter or longer. The MPLP, being a compromise between the renewal process and the NHPP, is such a model.

Results of goodness-of-fit tests reject the Weibull process as a choice for modeling duration dependence of bull and bear markets. The MPLP overcomes the shortcomings of the Weibull renewal process and is a more powerful model for dependence in financial cycles.

The results indicate negative duration dependence in all samples for bull markets and positive duration dependence in complete, peace time, and post-WWII samples of bear markets. There is no evidence of structural change after WWII in either bull or bear markets, with the exception that bear markets seem to have negative duration dependence before WWII and positive duration dependence after. Results also indicate that both bull and bear markets tend to get shorter over the long term, with some evidence that the rates are different in the postWWII period and after excluding war time cycles.

\section{Reference}

[1] J. E. Payne and T. W. Zuehlke, "Duration Dependence in Real Estate Investment Trusts,” Applied Financial Economics, Vol. 16, No. 5, 2006, pp. 413-423. doi:10.1080/09603100500391099

[2] S. J. Cochran and R. H. Defina, "Duration Dependence in the US Stock Market Cycle: A Parametric Approach,” Applied Financial Economics, Vol. 5, No. 5, 1995, pp. 27-37. doi:10.1080/758522757

[3] D. E. Sichel, "Business Cycle Duration Dependence: A Parametric Approach," The Review of Economics and Statistics, Vol. 73, No. 2, 1991, pp. 254-260. doi:10.2307/2109515

[4] H. Zhou and S. E. Rigdon, "Duration Dependence in U.S. 
Business Cycles: An Analysis Using the Modulated Power Law Process,” Journal of Economics and Finance, Vol. 32, No. 1, 2008, pp. 25-34. doi:10.1007/s12197-007-9005-3

[5] M. Berman, "Inhomogeneous and Modulated Gamma Processes,” Biometrika, Vol. 68, No. 1, 1982, pp. 142152.

[6] M. J. Lakey and S. E. Rigdon, "The Modulated Power Law Process," Proceedings of the 45th Annual Quality Congress, Milwaukee, 20-22 May 1991, pp. 559-563.

[7] S. E. Black and S. E. Rigdon, "Statistical Inference for a Modulated Power Law Process," Journal of Quality Technology, Vol. 28, No. 1, 1996, pp. 81-90.

[8] R. Frisch, "Propagation and Impulse Problems in Dynamic Economics," Economic Essays in Honor of Gustav Cassel, Allen and Unwin, London, 1933, pp. 171-173, 181-190, 197-203.

[9] E. Slutsky, "The Summation of Random Causes as the Source of Cyclic Processes,” Econometrica, Vol. 5, No. 2, 1937, pp. 105-146. doi:10.2307/1907241
[10] S. Chatterjee, "From Cycles to Shocks: Progress in Business-Cycle Theory,” 2000.

http://www.phil.frb.org/files/br/brma00sc.pdf

[11] B. S. Bernanke, M. Gertler and S. Gilchrist, “The Financial Accelerator in a Quantitative Business Cycle Framework," Review of Economics and Statistics, Vol. 78, No. 1, 1996, pp. 1-15.

[12] C. Nolan and C. Thoenissen, "Financial Shocks and the US Business Cycle," Journal of Monetary Economics, Vol. 56, No. 4, 2009, pp. 596-604. doi:10.1016/j.jmoneco.2009.03.007

[13] A. R. Pagan and K. A. Sossounov, “A Simple Framework for Analysing Bull and Bear Markets,” Journal of Applied Econometrics, Vol. 18, No. 1, 2003, pp. 23-46. doi:10.1002/jae.664

[14] M. A. Stephens, “Tests Based on EDF Statistics,” In: R. B. D’Agostino and M. A. Stephens, Eds., Goodness-of-Fit Techniques, Marcel Dekker, New York, 1986, pp. 97-193. 